\title{
On the Bounded Height Conjecture
}

\section{P. Habegger \\ ETH Zürich, Departement Mathematik, Rämistrasse 101, 8092 Zürich, Switzerland}

Correspondence to be sent to: habegger@math.ethz.ch

We prove the Bounded Height Conjecture formulated by Bombieri, Masser, and Zannier: given an irreducible closed subvariety $X \subset \mathrm{G}_{m}^{n}$, and after replacing $X$ by a natural and Zariski open subset; the set of its points contained in the union of all algebraic subgroups of codimension at least $\operatorname{dim} X$ has bounded absolute Weil height. We proceed to show some finiteness results related to conjectures stated by Zilber and Pink, if the codimension of the subgroups is at least $1+\operatorname{dim} X$.

\section{Introduction}

Let $G=\mathrm{G}_{m}^{n}$ be the algebraic torus and let $X \subset G$ be an irreducible closed subvariety defined over $\overline{\mathbf{Q}}$, an algebraic closure of $\mathbf{Q}$. In connection with the study of unlikely intersections of subvarieties of $G$ with algebraic subgroups, Bombieri, Masser, and Zannier [4] stated the so-called Bounded Height Conjecture: the set of points in a natural and Zariski open subset of $X$ contained in the union of all algebraic subgroups of codimension at least $\operatorname{dim} X$ has bounded height. The height involved is the absolute logarithmic Weil height (see Section 2). The natural subset is defined later.

The purpose of this paper is to prove the Bounded Height Conjecture for subvarieties of arbitrary dimension. 
Let $s$ be an integer, we set

$$
G^{[s]}=\bigcup_{\operatorname{codim} H \geq s} H(\overline{\mathbf{Q}})
$$

where the union runs over all algebraic subgroups $H \subset G$ of codimension at least $s$.

The Bounded Height Conjecture is related to conjectures stated independently by Zilber [21] and Pink [16] in the more general context of semiabelian varieties and mixed Shimura varieties, respectively. In the language of algebraic tori, these authors expect the following conjecture (in fact, it can be formulated more generally for varieties defined over $\mathbf{C}$, the field of complex numbers).

Conjecture 1.1. Let $X \subset \mathbf{G}_{m}^{n}$ be an irreducible closed subvariety defined over $\overline{\mathbf{Q}}$, which is not contained in a proper algebraic subgroup of $\mathrm{G}_{m}^{n}$. Then $X(\overline{\mathbf{Q}}) \cap G^{[1+\operatorname{dim} X]}$ is not Zariski dense in $X$.

In order to introduce the aforementioned natural Zariski open subset of $X$, we assume for the moment that our variety is defined over C. For a possibly reducible variety $Y$ containing a point $p$, we let $\operatorname{dim}_{p} Y$ denote the largest dimension of an irreducible component of $Y$ passing through $p$. For an integer $s$, we define $X^{\mathrm{oa},[s]}$ to be $X(\mathbf{C})$ deprived of all $p \in X(\mathbf{C})$ such that $\operatorname{dim}_{p} X \cap p H \geq \max \{1, s+\operatorname{dim} H-n+1\}$ for some algebraic subgroup $H \subset G$. This definition is interesting only when $s \geq \operatorname{dim} X$, otherwise, we immediately see $X^{\mathrm{oa},[s]}=\emptyset$, if $X$ has a positive dimension (consider $H=G$ ). Bombieri, Masser, and Zannier [4] proved that $X^{\mathrm{oa}}=X^{\mathrm{oa},[\operatorname{dim} X]}$ is Zariski open in $X$ (but possibly empty) and even showed a structure theorem. They then generalized their results in Lemma 5 [6] and showed that $X^{\mathrm{oa},[s]}$ is Zariski open in $X$. It also follows from their work that if $X$ is defined over $\overline{\mathbf{Q}}$, then so is the complement of $X^{\mathrm{oa},[s]}$; in this situation we will usually identify $X^{\mathrm{oa},[s]}$ with $X^{\mathrm{oa},[s]} \cap X(\overline{\mathbf{0}})$.

In the abelian case, related sets were defined and studied by Rémond [17]. His description of $Z_{X \text {,an }}^{(s+1)}$ translates without difficulty to the toric setting and in fact we have $X^{\mathrm{oa},[s]}=X \backslash Z_{X, \mathrm{an}}^{(s+1)}$.

Our main result gives a height bound not only for points on varieties contained in certain algebraic subgroups, but also for points near such subgroups with respect to the absolute logarithmic Weil height $h$. If $S$ is any subset of $G(\overline{\mathbf{0}})$ and $\epsilon \geq 0$, we define the truncated cone around $S$ as

$$
\mathcal{C}(S, \epsilon)=\{p \in G(\overline{\mathbf{Q}}) ; p=a b \text { with } a \in S \text { and } b \in G(\overline{\mathbf{0}}) \text { where } h(b) \leq \epsilon(1+h(a))\} \text {. }
$$

We note that a torsion point of $G(\overline{\mathbf{0}})$ has height zero, therefore $\mathcal{C}(S, \epsilon)$ always contains $S$. 
Theorem 1.2. Let $G=\mathrm{G}_{m}^{n}$, let $X \subset G$ be an irreducible closed subvariety defined over $\overline{\mathbf{Q}}$, and let $s$ be an integer. There exists an $\epsilon>0$ such that the height is bounded from above on $X^{\mathrm{oa},[s]} \cap \mathcal{C}\left(G^{[s]}, \epsilon\right)$.

If we take $s=\operatorname{dim} X$, the theorem above implies the following statement, which was conjectured in [4].

Corollary 1.3 Bounded Height Conjecture. Let $G=\mathrm{G}_{m}^{n}$ and let $X \subset G$ be an irreducible closed subvariety defined over $\overline{\mathbf{0}}$. Then the height is bounded from above on $X^{\mathrm{oa}} \cap G^{[\mathrm{dim} X]}$.

The codimension of the involved subgroups is best possible, i.e., it is minimal as a function of $\operatorname{dim} X$, as soon as $X^{\mathrm{oa}} \neq \emptyset$. In this case the codimension cannot be reduced even after replacing $X^{\text {oa }}$ by any nonempty Zariski open subset of $X$ (see Proposition 1 [13]).

Certain instances of this conjecture already exist in the literature. For example, Bombieri, Masser, and Zannier handled the case of curves [3] and planes [5]. The case of hypersurfaces, i.e., subvarieties of $G$ of codimension 1, was resolved by Bombieri and Zannier [20] even before the case of curves.

In Theorem 1 [13], the author derived an effective and explicit height bound for $X^{\text {oa }} \cap G^{[s]}$ under the stronger hypothesis $s>n-n / \operatorname{dim} X$. This result only implies the corollary above if $X$ is a curve or hypersurface.

The proof of Theorem 1.2 relies on a theorem of Ax (see Section 7) and intersection theory; thus, it differs from the approach in [13]. Intersection theory forces us to work in compactifications of $\mathbf{G}_{m}^{n}$ which will depend on a fixed morphism $\mathbf{G}_{m}^{n} \rightarrow \mathbf{G}_{m}^{r}$. On the other hand, we have at our disposal Siu's theorem, which guarantees the existence of a nonzero global section of a certain line bundle under a numerical criterion on intersection numbers. The lack of a suitable theorem of the cube for algebraic tori is compensated by a multiprojective version of Bézout's theorem due to Philippon. Further ingredients include elementary results from diophantine approximation, geometry of numbers, and a simple compactness argument (see Lemma 6.3). The latter renders the proof ineffective from a formal point of view, but for the moment we are only interested in the existence of a height upper bound. Nevertheless, an effective version of Theorem 1.2 using different methods should not be ruled out and is a possible subject of future work.

We note that the abelian analog to Theorem 1.2 also holds and is demonstrated in another paper [12]. Of course, the difficulty concerning compactifications of the ambient 
algebraic group disappears. On the other hand, algebraic subgroups become more difficult to describe; it is the fact that an abelian variety may not have an algebraic subgroup of any possible dimension which causes most problems.

In the presence of height bounds, it has been known since the work of Bombieri, Masser, and Zannier [3] on curves that Lehmer-type height lower bounds, together with algebraic number theory, can lead to finiteness results on the intersection of subvarieties of $G$ with $G^{[s]}$. In the meantime, a semirelative Lehmer-type height lower bound has been proved by Amoroso and David [1]. This lower bound leads to a simplification in finiteness proofs for curves and facilitates them for arbitrary varieties. As a result of Theorem 1.2 and Bombieri, Masser, and Zannier (see Theorem 8.3 in Section 8), we obtain the following corollary.

Corollary 1.4. Let $G=\mathrm{G}_{m}^{n}$ and let $X \subset G$ be an irreducible closed subvariety defined over $\overline{\mathbf{Q}}$, then $X^{\text {oa }} \cap G^{[1+\operatorname{dim} X]}$ is finite.

Even though $1+\operatorname{dim} X$ cannot be replaced by anything smaller in this corollary, the set $X^{\text {oa }}$ is possibly not optimal. It was conjectured by Bombieri, Masser, and Zannier (torsion finiteness conjecture [4]) that finiteness holds in Corollary 1.4 when working over C and with $X^{\text {oa }}$ replaced by the possibly larger $X^{\text {ta }}$. We will not define this subset of $X(\mathbf{C})$ here; it is also conjectured [4] to be Zariski open in $X$. The connection between these two conjectures and the ones by Zilber and Pink for the algebraic torus described further up has been discussed by Bombieri, Masser, and Zannier [6].

The next corollary shows that Conjecture 1.1 holds for sufficiently generic subvarieties of $\mathbf{G}_{m}^{n}$.

Corollary 1.5. Let $X \subset \mathbf{G}_{m}^{n}$ be an irreducible closed subvariety defined over $\overline{\mathbf{Q}}$ with coordinate functions $x_{1}, \ldots, x_{n}$ considered as elements of the function field of $X$. Suppose that for all $(\operatorname{dim} X)$-tuples of $\mathbf{Z}$-linearly independent vectors $\left(u_{i 1}, \ldots, u_{i n}\right) \in \mathbf{Z}^{n}$, the functions

$$
x_{1}^{u_{i 1}} \cdots x_{n}^{u_{i n}} \quad(1 \leq i \leq \operatorname{dim} X)
$$

are algebraically independent over $\overline{\mathbf{0}}$. Then Conjecture 1.1 holds for $X$.

This paper is structured as follows. Heights and notation are introduced in Section 2. In Section 3, we construct a suitable compactification of $\mathbf{G}_{m}^{n}$ with respect to a fixed morphism $\mathbf{G}_{m}^{n} \rightarrow \mathbf{G}_{m}^{r}$. Diophantine approximation is applied in Section 4 to derive an upper bound for a height, which we proceed to bound from below in Section 5 using 
Siu's theorem. In Section 6, we count periods of $\mathbf{G}_{m}^{n}$ using geometry of numbers in order to apply Ax's theorem in Section 7; this enables us to refine the lower bound from Section 5. Finally, the proof of Theorem 1.2 is completed in Section 8 where we also prove Corollaries 1.4 and 1.5.

\section{Notation and Heights}

Let $r$ and $n$ be integers with $1 \leq r \leq n$. We may identify the group of homomorphisms of algebraic groups $\mathbf{G}_{m}^{n} \rightarrow \mathbf{G}_{m}^{r}$ with $\operatorname{Mat}_{r n}(\mathbf{Z})$, the group of $r \times n$ matrices in integer coefficients. Given $p=\left(p_{1}, \ldots, p_{n}\right)$ with nonzero coordinates in some field and $u=\left(u_{1}, \ldots, u_{n}\right) \in \mathbf{Z}^{n}$, we set $p^{u}=p_{1}^{u_{1}} \cdots p_{n}^{u_{n}}$.

We let $|\cdot|$ denote the euclidean or hermitian norm on $\operatorname{Mat}_{r n}(\mathbf{R})$ or $\operatorname{Mat}_{r n}(\mathbf{C})$, respectively.

We continue by defining the absolute logarithmic Weil height, or height for short, on algebraic points of $n$-dimensional projective space $\mathbf{P}^{n}$. For any place $v$ of a number field $K$, we let $K_{v}$ denote the completion of $K$ with respect to $v$. If $p=\left[p_{0}: \cdots: p_{n}\right] \in \mathbf{P}^{n}(\overline{\mathbf{Q}})$ with $p_{i} \in K$ for all $i$ we define the projective height of $p$ as

$$
h(p)=\frac{1}{[K: \mathbf{0}]} \sum_{v}\left[K_{v}: \mathbf{Q}_{v}\right] \log \max \left\{\left|p_{0}\right|_{v}, \ldots,\left|p_{n}\right|_{v}\right\}
$$

where the sum runs over all places of $K$ normalized such that they restrict to the usual complex, respectively $p$-adic absolute values on $\mathbf{0}$. Hence, the product formula holds and our height is independent of the choice of projective coordinates of $p$ in $K$. Furthermore, it is well known that $h(p)$ is independent of the choice of a number field $K$ containing the $p_{i}$. Therefore, the height is defined on $\mathbf{P}^{n}(\overline{\mathbf{Q}})$; it is also nonnegative since we can choose one projective coordinate of $p$ to be 1. Details are contained in Sections 1.4 and 1.5 [7].

There is an open immersion $\mathbf{G}_{m}^{n} \rightarrow \mathbf{P}^{n}$ which sends a point $\left(p_{1}, \ldots, p_{n}\right)$ to [1: $p_{1}: \cdots: p_{n}$ ]. By abuse of notation we define the height $h(p)$ of $p \in \mathbf{G}_{m}^{n}(\overline{\mathbf{Q}})$ as the height of the image of $p$ with respect to this immersion. From the local nature of our definition of the height, we derive $\max \left\{h\left(p_{1}\right), \ldots, h\left(p_{n}\right)\right\} \leq h(p) \leq h\left(p_{1}\right)+\cdots+h\left(p_{n}\right)$.

Our height interacts nicely with the group structure on $\mathbf{G}_{m}^{n}(\overline{\mathbf{Q}})$. Indeed, if $p, q \in \mathbf{G}_{m}^{n}(\overline{\mathbf{Q}})$ then $h(p q) \leq h(p)+h(q)$. Moreover, if $k$ is a nonnegative integer then the height is homogeneous in the sense that $h\left(p^{k}\right)=k h(p)$. If $k$ is any integer, then a consequence of the product formula is $h\left(p^{k}\right) \leq n|k| h(p)$ and if $n=1$, then $h\left(p^{k}\right)=|k| h(p)$. 
For the rest of this section all varieties are assumed to be defined over $\overline{\mathbf{0}}$. We will also need the notion of a height $h_{X, \mathcal{L}}$ associated to a line bundle $\mathcal{L}$ on an irreducible projective variety $X$ (see Theorem 2.3.8 [7]). The association $\mathcal{L} \mapsto h_{X, \mathcal{L}}$ satisfies certain useful functional properties in connection with the Picard group of $X$, the group of isomorphism classes of line bundles on $X$. In fact, we will usually identify a line bundle with its isomorphism class. Actually, $h_{X, \mathcal{L}}$ is not an honest function $X(\overline{\mathbf{Q}}) \rightarrow \mathbf{R}$, but an equivalence class of such where two are called equivalent if their difference is bounded on $X(\overline{\mathbf{Q}})$. Since it is not the purpose of this paper to obtain explicit height bounds, we fix once and for all a representative of each class and denote it with $h_{X, \mathcal{L}}$. The association $\mathcal{L} \mapsto h_{X, \mathcal{L}}$ still satisfies functional properties, but only up to a bounded term. Explicitly, let $\mathcal{M}$ be a second line bundle on $X$, then the two functions $h_{X, \mathcal{L} \otimes \mathcal{M}}$ and $h_{X, \mathcal{L}}+h_{X, \mathcal{M}}$ differ by a bounded function on $X(\overline{\mathbf{Q}})$. Furthermore, if $Y$ is another irreducible projective variety and $f: Y \rightarrow X$ is a morphism, then $h_{Y, f^{*} \mathcal{L}}$ and $h_{X, \mathcal{L}} \circ f$ differ by a bounded function. If we assume that $\mathcal{L}$ has a nonzero global section, then there exists a Zariski open and nonempty $U \subset X$ such that $h_{X, \mathcal{L}}$ is bounded from below on $U(\overline{\mathbf{Q}})$. Finally, the height is gauged in the following way: let $\mathcal{O}(1)$ be the dual of the tautological line bundle on $\mathbf{P}^{n}$, then $h_{\mathbf{P}^{n}, \mathcal{O}(1)}$ and the absolute logarithmic Weil height $h$ differ by a bounded function.

\section{Compactifying $G_{m}^{n}$}

This section provides a compactification of $\mathbf{G}_{m}^{n}$, which is compatible with a fixed homomorphism of algebraic groups $\varphi: \mathbf{G}_{m}^{n} \rightarrow \mathbf{G}_{m}^{r}$ where $1 \leq r \leq n$. The notation and the properties described below are used throughout the paper.

We set

$$
\Gamma(\varphi) \subset \mathbf{G}_{m}^{n} \times \mathbf{G}_{m}^{r}
$$

to be the graph of $\varphi$. The product of the open immersions $\mathbf{G}_{m}^{n} \rightarrow \mathbf{P}^{n}$ and $\mathbf{G}_{m}^{r} \rightarrow \mathbf{P}^{r}$ described in Section 2 gives an open immersion $\mathbf{G}_{m}^{n} \times \mathbf{G}_{m}^{r} \rightarrow \mathbf{P}^{n} \times \mathbf{P}^{r}$. We will consider $\mathbf{G}_{m}^{n} \times \mathbf{G}_{m}^{r}$ as contained in $\mathbf{P}^{n} \times \mathbf{P}^{r}$ with respect to this immersion. We let $\overline{\Gamma(\varphi)}$ be the Zariski closure in $\mathbf{P}^{n} \times \mathbf{P}^{r}$ of $\Gamma(\varphi)$, it is clearly an irreducible projective variety and we have $\overline{\Gamma(\varphi)} \cap\left(\mathbf{G}_{m}^{n} \times \mathbf{G}_{m}^{r}\right)=\Gamma(\varphi)$. We also define $b$ as the composition of $\mathbf{G}_{m}^{n} \rightarrow \Gamma(\varphi)$ given by $p \mapsto(p, \varphi(p))$ with the inclusion $\Gamma(\varphi) \rightarrow \overline{\Gamma(\varphi)}$. Let $\pi_{1,2}$ be the projections from $\mathbf{P}^{n} \times \mathbf{P}^{r}$ to $\mathbf{P}^{n}$ and $\mathbf{P}^{r}$, respectively, and let $\pi=\left.\pi_{1}\right|_{\overline{\Gamma(\varphi)}}$ and $\bar{\varphi}=\left.\pi_{2}\right|_{\overline{\Gamma(\varphi)}}$, these are both proper morphisms. 
We have the following commutative diagram:

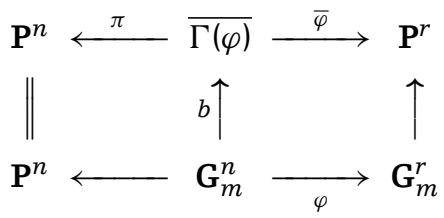

Now let $X \subset \mathbf{G}_{m}^{n}$ be an irreducible closed subvariety of dimension $r$ defined over C. It will be useful to denote $\bar{X}$ the Zariski closure of $X$ in $\mathbf{P}^{n}$ with respect to the open immersion $\mathbf{G}_{m}^{n} \rightarrow \mathbf{P}^{n}$, then $\left(\bar{X} \times \mathbf{P}^{r}\right) \cap\left(\mathbf{G}_{m}^{n} \times \mathbf{G}_{m}^{r}\right)=X \times \mathbf{G}_{m}^{r}$. Let $\bar{X}^{\varphi}$ be the Zariski closure of $b(X)$ in $\overline{\Gamma(\varphi)} \subset \mathbf{P}^{n} \times \mathbf{P}^{r}$. Then $\bar{X}^{\varphi}$ is an irreducible projective variety, and so $\left.\pi\right|_{\bar{X}^{\varphi}}$ and $\left.\bar{\varphi}\right|_{\bar{X}^{\varphi}}$ are proper morphisms. We have the following commutative diagram:

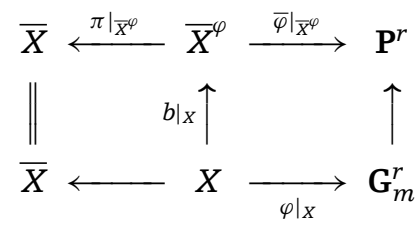

For an irreducible variety $Y$ and an irreducible closed subvariety $Z \subset Y$, we let [Z] denote the equivalence class of cycles on $Y$ rationally equivalent to $Z$. Furthermore, $c_{1}(\mathcal{L})$ is the first Chern class of a line bundle $\mathcal{L}$ on $Y$ (see Chapters 1 and 2 [10]).

Let $Y$ and $Z$ be any irreducible varieties and $f: Y \rightarrow Z$ be a proper morphism. If $f$ is dominant, then the function field of $Z$ is via $f$ naturally a subfield of the function field of $Z$. If $\operatorname{dim} Y=\operatorname{dim} Z$ the degree of $f$, denoted by $\operatorname{deg}(f)$, is the degree of this (finite) field extension. If $\operatorname{dim} Y>\operatorname{dim} Z$ we set $\operatorname{deg}(f)=0$. If $f$ is not dominant we also set $\operatorname{deg}(f)=0$.

We define

$$
\Delta_{X}(\varphi)=\operatorname{deg}\left(\left.\bar{\varphi}\right|_{\bar{X}}\right)
$$

so $\Delta_{X}$ is nonnegative. We will often abbreviate $\Delta_{X}$ with $\Delta$.

We collect some facts on $\Delta$ in the next lemma.

Lemma 3.1. The function $\Delta_{X}$ satisfies the following properties:

(i) We have $\Delta_{X}(\varphi)=\left(c_{1}\left(\bar{\varphi}^{*} \mathcal{O}(1)\right)^{r}\left[\bar{X}^{\varphi}\right]\right)$.

(ii) There exists $U \subset \mathbf{G}_{m}^{r}$ Zariski open and nonempty such that the fiber of $\left.\varphi\right|_{X}$ : $X \rightarrow \mathbf{G}_{m}^{r}$ above any point of $U$ has cardinality equal to $\Delta_{X}(\varphi)$. 
(iii) There exists a function $\operatorname{Mat}_{r n}(\mathbf{0}) \rightarrow[0, \infty)$ which extends $\Delta_{X}$ and which we also denote with $\Delta_{X}$ such that $\Delta_{X}(\lambda \varphi)=|\lambda|^{r} \Delta_{X}(\varphi)$ for all $\lambda \in \mathbf{Q}$ and $\varphi \in \operatorname{Mat}_{r n}(\mathbf{Q})$.

Proof. For dominant $\left.\bar{\varphi}\right|_{\bar{X}^{\varphi}}$ the projection formula (see Chapter 2 [10]) implies

$$
\Delta(\varphi)=\operatorname{deg}\left(\left.\bar{\varphi}\right|_{\bar{X}^{\varphi}}\right)=\operatorname{deg}\left(\left.\bar{\varphi}\right|_{\bar{X}^{\varphi}}\right)\left(C_{1}(\mathcal{O}(1))^{r}\left[P^{r}\right]\right)=\left(C_{1}\left(\bar{\varphi}^{*} \mathcal{O}(1)\right)^{r}\left[\bar{X}^{\varphi}\right]\right),
$$

equality also holds if $\left.\bar{\varphi}\right|_{\bar{X}^{\varphi}}$ is not dominant since then both sides vanish. Part (i) of the lemma follows.

Generically, the two morphisms $\left.\varphi\right|_{X}: X \rightarrow \mathbf{G}_{m}^{r}$ and $\left.\bar{\varphi}\right|_{\bar{X}^{\varphi}}: \bar{X}^{\varphi} \rightarrow \mathbf{P}^{r}$ have fibers of equal cardinality because the second and third vertical morphisms in (2) are birational. To show part (ii), we may assume that $\left.\varphi\right|_{X}$ is dominant, and hence has generically finite fibers. After restricting domain and target of $\left.\varphi\right|_{X}$ to suitable Zariski open nonempty subsets, we obtain a finite and hence proper morphism whose fiber above any point of a Zariski open and nonempty subset of $\mathbf{G}_{m}^{n}$ is equal to the corresponding fiber of $\left.\varphi\right|_{X}$. Since this restriction of $\left.\varphi\right|_{X}$ is proper, it makes sense to speak of its degree which equals $\Delta(\varphi)$. As we are in characteristic zero, the restriction is unramified above a Zariski open dense subset of the target. The fibers above points of this set have cardinality $\Delta(\varphi)$.

The morphism that takes a point of $\mathbf{G}_{m}^{r}$ to its $\lambda$-th power has fibers of cardinality exactly $|\lambda|^{r}$ if $\lambda \neq 0$. This and the second claim of the lemma imply that $\Delta(\lambda \varphi)=|\lambda|^{r} \Delta(\varphi)$ for all $\lambda \in \mathbf{Z}$ and $\varphi \in \operatorname{Mat}_{r n}$ (Z). Part (iii) now follows easily.

It is natural to hope to extend $\Delta$ to a continuous function $\operatorname{Mat}_{r n}(\mathbf{R}) \rightarrow[0, \infty)$. Some parts of the proof of Theorem 1.2 would simplify if this were possible. Moreover, a precise knowledge of $\Delta$ may even render the application of Ax's theorem in Section 7 obsolete. But, in this paper we are content with $\Delta$ being defined only on $\operatorname{Mat}_{r n}(\mathbf{Q})$.

We proceed by bounding from above a certain intersection number related to the one showing up in Lemma 3.1. Our main tool is a Bézout theorem for a product of projective spaces due to Philippon. For us, it suffices to consider the product of two projective spaces. For an irreducible closed subvariety $V \subset \mathbf{P}^{n} \times \mathbf{P}^{r}$, we set

$$
H(V ; d, e)=(\operatorname{dim} V) ! \sum_{i_{1}+i_{2}=\operatorname{dim} V}\left(c_{1}\left(\pi_{1}^{*} \mathcal{O}(1)\right)^{i_{1}} C_{1}\left(\pi_{2}^{*} \mathcal{O}(1)\right)^{i_{2}}[V]\right) \frac{d^{i_{1}} e^{i_{2}}}{i_{1} ! i_{2} !}
$$


here the sum runs over integers $i_{1}, i_{2}$ with $0 \leq i_{1} \leq n, 0 \leq i_{2} \leq r$. Then $H(V ; d, e)$, when considered as polynomial in $d$ and $e$, is a multiple of the highest degree homogeneous part of the Hilbert polynomial of $V$.

Theorem 3.2 (Philippon). Let $V$ be an irreducible subvariety of $\mathbf{P}^{n} \times \mathrm{P}^{r}$ and let $\mathcal{F}$ be a set of bihomogeneous polynomials of bidegree bounded by $(d, e)$. Then

$$
\sum_{Z} H(Z ; d, e) \leq H(V ; d, e)
$$

where the sum runs over all irreducible components $Z$ of the intersection of $V$ with the set of common zeros of all polynomials in $\mathcal{F}$.

Proof. The bound (4) is a consequence of the first statement of Proposition 3.3 [15] where we take $I_{0}$ to be the ideal of $V$.

We let $\operatorname{deg}(X)$ denote the degree of $\bar{X} \subset \mathbf{P}^{n}$ with respect to $\mathcal{O}(1)$, i.e., $\operatorname{deg}(X)=$ $\left(c_{1}(\mathcal{O}(1))^{r}[\bar{X}]\right)$.

Lemma 3.3. In the above notation, we have

$$
\left(c_{1}\left(\pi^{*} \mathcal{O}(1)\right) C_{1}\left(\bar{\varphi}^{*} \mathcal{O}(1)\right)^{r-1}\left[\bar{X}^{\varphi}\right]\right) \leq C_{1}|\varphi|^{r-1}
$$

with $C_{1}=(4 n)^{r} \operatorname{deg}(X)$.

Proof. This is an application of Philippon's theorem; we take $\mathcal{F}$ to be the set of polynomials $f_{1}, \ldots, f_{r}$ defined in the following manner: say $\varphi$ has rows $u_{1}, \ldots, u_{r} \in \mathbf{Z}^{n}$ with $u_{i}=\left(u_{i 1}, \ldots, u_{i n}\right)$ for $1 \leq i \leq r$. We set

$$
\delta_{i}=\max \left\{\sum_{j=1}^{n} \max \left\{0, u_{i j}\right\}, \sum_{j=1}^{n} \max \left\{0,-u_{i j}\right\}\right\}
$$

and

$$
f_{i}=Y_{0} X_{0}^{\delta_{i}} \prod_{j=1}^{n} X_{0}^{-\max \left\{0, u_{i j}\right\}} X_{i}^{\max \left\{0, u_{i j}\right\}}-Y_{i} X_{0}^{\delta_{i}} \prod_{j=1}^{n} X_{0}^{-\max \left\{0,-u_{i j}\right\}} X_{i}^{\max \left\{0,-u_{i j}\right\}}
$$


for each $1 \leq i \leq r$, here $X_{0}, \ldots, X_{n}$ are the homogeneous coordinates of $\mathbf{P}^{n}$ and $Y_{0}, \ldots, Y_{r}$ are the homogeneous coordinates of $\mathbf{P}^{r}$. The $\delta_{i}$ are chosen such that each $f_{i}$ is a bihomogeneous polynomial of bidegree $\left(\delta_{i}, 1\right)$. Say $d=\max \left\{\delta_{1}, \ldots, \delta_{r}\right\}$ and $e=1$, so $(d, e)$ bounds the bidegree of each $f_{i}$.

Let $Y$ denote the set of common zeros of all $f_{i}$ in $\mathbf{P}^{n} \times \mathbf{P}^{r}$. It follows from the definition of $Y$ that $Y \cap\left(\mathbf{G}_{m}^{n} \times \mathbf{G}_{m}^{r}\right)=\Gamma(\varphi)=\overline{\Gamma(\varphi)} \cap\left(\mathbf{G}_{m}^{n} \times \mathbf{G}_{m}^{r}\right)$. So $\left(\bar{X} \times \mathbf{P}^{r}\right) \cap Y$ and $\left(\bar{X} \times \mathbf{P}^{r}\right) \cap$ $\overline{\Gamma(\varphi)}$ coincide on the Zariski open set $\mathbf{G}_{m}^{n} \times \mathbf{G}_{m}^{r} \subset \mathbf{P}^{n} \times \mathbf{P}^{r}$ and equal $\left(X \times \mathbf{G}_{m}^{r}\right) \cap \Gamma(\varphi)=b(X)$ there. Hence $\bar{X}^{\varphi}$ is an irreducible component of $\left(\bar{X} \times \mathbf{P}^{r}\right) \cap Y$. We conclude that $H\left(\bar{X}^{\varphi} ; d, e\right)$ is a term in the right-hand side of (4) when $V=\bar{X} \times \mathbf{P}^{r}$.

It is well known that the coefficients of the homogeneous Hilbert polynomial defined in (3) are nonnegative; in particular $H(Z ; d, e) \geq 0$ for admissible $Z$. By Theorem 3.2 we get $H\left(\bar{X}^{\varphi} ; d, e\right) \leq H\left(\bar{X} \times \mathbf{P}^{r} ; d, e\right)$ and even

$$
r\left(C_{1}\left(\pi^{*} \mathcal{O}(1)\right) C_{1}\left(\bar{\varphi}^{*} \mathcal{O}(1)\right)^{r-1}\left[\bar{X}^{\varphi}\right]\right) d e^{r-1} \leq H\left(\bar{X} \times \mathbf{P}^{r} ; d, e\right)
$$

where the left-hand side is the term of $H\left(\bar{X}^{\varphi} ; d, e\right)$ corresponding to $i_{1}=1$ and $i_{2}=r-1$. We proceed by evaluating the right-hand side of (5). By definition

$$
H\left(\bar{X} \times \mathbf{P}^{r} ; d, e\right)=(2 r) ! \sum_{i_{1}+i_{2}=2 r}\left(c_{1}\left(\pi_{1}^{*} \mathcal{O}(1)\right)^{i_{1}} c_{1}\left(\pi_{2}^{*} \mathcal{O}(1)\right)^{i_{2}}\left[\bar{X} \times \mathbf{P}^{r}\right]\right) \frac{d^{i_{1}} e^{i_{2}}}{i_{1} ! i_{2} !},
$$

where the sum runs over all integers $i_{1}, i_{2}$ with $0 \leq i_{1} \leq n$ and $0 \leq i_{2} \leq \mathrm{r}$. Commutativity and the projection formula give

$$
\left(c_{1}\left(\pi_{1}^{*} \mathcal{O}(1)\right)^{i_{1}} c_{1}\left(\pi_{2}^{*} \mathcal{O}(1)\right)^{i_{2}}\left[\bar{X} \times \mathbf{P}^{r}\right]\right)=\left(c_{1}(\mathcal{O}(1))^{i_{2}} \pi_{2 *}\left(c_{1}\left(\pi_{1}^{*} \mathcal{O}(1)\right)^{i_{1}}\left[\bar{X} \times \mathbf{P}^{r}\right]\right)\right) .
$$

If $i_{1}>\operatorname{dim} \bar{X}=r$, then $c_{1}\left(\pi_{1}^{*} \mathcal{O}(1)\right)^{i_{1}}\left[\bar{X} \times \mathbf{P}^{r}\right]$ is the class of cycles on $\mathbf{P}^{n} \times \mathbf{P}^{r}$ linearly equivalent to zero. Hence, the intersection number above vanishes. We conclude that all terms on the right in (6) except possibly the one corresponding to $i_{1}=i_{2}=r$ are 0 , and so

$$
H\left(\bar{X} \times \mathbf{P}^{r} ; d, e\right)=\frac{(2 r) !}{(r !)^{2}}\left(c_{1}\left(\pi_{1}^{*} \mathcal{O}(1)\right)^{r} c_{1}\left(\pi_{2}^{*} \mathcal{O}(1)\right)^{r}\left[\bar{X} \times \mathbf{P}^{r}\right]\right)(d e)^{r}
$$


The intersection number on the right depends only on $X$. Finally, we can evaluate it using the projection formula,

$$
\begin{aligned}
\left(C_{1}\left(\pi_{1}^{*} \mathcal{O}(1)\right)^{r} C_{1}\left(\pi_{2}^{*} \mathcal{O}(1)\right)^{r}\left[\bar{X} \times \mathbf{P}^{r}\right]\right) & =\left(c_{1}(\mathcal{O}(1))^{r} \pi_{1 *}\left(C_{1}\left(\pi_{2}^{*} \mathcal{O}(1)\right)^{r}\left[\bar{X} \times \mathbf{P}^{r}\right]\right)\right) \\
& =\left(c_{1}(\mathcal{O}(1))^{r}[\bar{X}]\right) \\
& =\operatorname{deg}(X) .
\end{aligned}
$$

Hence,

$$
H\left(\bar{X} \times \mathbf{P}^{r} ; d, e\right)=\frac{(2 r) !}{(r !)^{2}} \operatorname{deg}(X)(d e)^{r} \leq 4^{r} \operatorname{deg}(X)(d e)^{r} .
$$

This inequality together with (5) gives

$$
\left(C_{1}\left(\pi^{*} \mathcal{O}(1)\right) C_{1}\left(\bar{\varphi}^{*} \mathcal{O}(1)\right)^{r-1}\left[\bar{X}^{\varphi}\right]\right) \leq 4^{r} \operatorname{deg}(X) d^{r-1} e .
$$

We conclude the proof because $e=1$, and since $\delta_{i} \leq \sqrt{n}|\varphi|$ and so $d \leq \sqrt{n}|\varphi|$.

\section{Auxiliary Morphism and Height Upper Bound}

We use a simple result from diophantine approximation to obtain a height upper bound in Lemma 4.4.

Let $s$ and $n$ be integers with $1 \leq s \leq n$.

Lemma 4.1. Let $Q>1$ be a real number and let $\varphi_{0} \in \operatorname{Mat}_{s n}(\mathbf{R})$, there exist $q \in \mathbf{Z}$ and $\varphi \in \operatorname{Mat}_{s n}(\mathbf{Z})$ such that

$$
1 \leq q \leq Q \quad \text { and } \quad\left|q \varphi_{0}-\varphi\right| \leq \frac{\sqrt{s n}}{Q^{1 /(s n)}}
$$

Proof. Let $\varphi_{i j}^{\prime}(1 \leq i \leq s, 1 \leq j \leq n)$ be the coefficients of $\varphi_{0}$. By Theorem 6 ([8], p. 13), there exist integers $q, \varphi_{i j}$ with $1 \leq q \leq Q$ and $\left|q \varphi_{i j}^{\prime}-\varphi_{i j}\right|<Q^{-1 /(s n)}$ for all $1 \leq i \leq s$ and $1 \leq j \leq n$. The lemma follows on taking $\varphi$ to be the matrix with coefficients $\varphi_{i j}$. 
We define $\mathcal{K}_{s n} \subset \operatorname{Mat}_{s n}(\mathbf{R})$ to be the compact set of all matrices whose rows are orthonormal. All elements of $\mathcal{K}_{s n}$ have rank $s$.

Lemma 4.2. Say $W \subset \operatorname{Mat}_{s n}(\mathbf{R})$ is an open neighborhood of $\mathcal{K}_{s n}$. There is $Q_{0} \geq 1$ (which may depend on $W$ ) with the following property. Let $Q>Q_{0}$ be a real number and let $\varphi_{0} \in \operatorname{Mat}_{s n}(\mathbf{R})$ with rank $s$, there exist $q \in \mathbf{Z}, \varphi \in \operatorname{Mat}_{s n}(\mathbf{Z})$, and $\theta \in \operatorname{Mat}_{s}(\mathbf{Q})$ such that

$$
1 \leq q \leq Q, \quad \frac{\varphi}{q} \in W, \quad\left|q \theta \varphi_{0}-\varphi\right| \leq \frac{\sqrt{s n}}{Q^{1 /(s n)}}, \quad \text { and } \quad|\varphi| \leq(s+1) q
$$

Proof. Since $\mathcal{K}_{s n}$ is compact, there exists $\epsilon>0$ with the following property: if $\varphi^{\prime} \in \mathcal{K}_{s n}$ and $\varphi^{\prime \prime} \in \operatorname{Mat}_{s n}(\mathbf{R})$ with $\left|\varphi^{\prime}-\varphi^{\prime \prime}\right|<\epsilon$ then $\varphi^{\prime \prime} \in W$. We may clearly assume $\epsilon \leq 1$. We choose $Q_{0}$ such that $\sqrt{s n} Q_{0}^{-1 /(s n)}=\epsilon / 2$, so $Q_{0} \geq 1$.

Let $\varphi_{0}$ be as in the hypothesis. The $\mathbf{R}$-vector space generated by the rows of $\varphi_{0}$ has dimension $s$ and admits an orthonormal basis. In other words, there exists an invertible $\theta_{0} \in \operatorname{Mat}_{s}(\mathbf{R})$ such that $\theta_{0} \varphi_{0} \in \mathcal{K}_{s n}$.

Since $\operatorname{Mat}_{s}(\mathbf{Q}) \subset \operatorname{Mat}_{s}(\mathbf{R})$ lies dense we may find $\theta \in \operatorname{Mat}_{s}(\mathbf{Q})$ with

$$
\left|\theta \varphi_{0}-\theta_{0} \varphi_{0}\right|<\frac{\epsilon}{2}
$$

Now say $Q>Q_{0}$. By Lemma 4.1, we approximate $\theta \varphi_{0}$ to get an integer $q$ with $1 \leq q \leq Q$ and $\varphi \in \operatorname{Mat}_{s n}(\mathbf{Z})$ such that

$$
\left|q \theta \varphi_{0}-\varphi\right| \leq \frac{\sqrt{s n}}{Q^{1 /(s n)}}
$$

In particular, the first three inequalities in (7) hold. Furthermore,

$$
\left|\theta \varphi_{0}-\frac{\varphi}{q}\right| \leq \frac{\sqrt{s n}}{Q^{1 /(s n)} q}<\frac{\sqrt{s n}}{Q_{0}^{1 /(s n)} q}=\frac{\epsilon}{2 q}
$$

This inequality and (8) as well as the triangle inequality and $q \geq 1 \mathrm{imply}\left|\theta_{0} \varphi_{0}-\varphi / q\right|<\epsilon$. So $\varphi / q \in W$ since $\theta_{0} \varphi_{0} \in \mathcal{K}_{s n}$.

To prove the last assertion in (7), we apply the triangle inequality to (8) and obtain $\left|\theta \varphi_{0}\right|<\left|\theta_{0} \varphi_{0}\right|+\epsilon / 2$. The rows of $\theta_{0} \varphi_{0} \in \mathcal{K}_{s n}$ have norm 1 , hence $\left|\theta_{0} \varphi_{0}\right|=\sqrt{s}$, and so

$$
\left|\theta \varphi_{0}\right|<\sqrt{s}+\frac{\epsilon}{2} \leq s+\frac{1}{2}
$$


The triangle inequality applied to (9) gives $|\varphi| \leq q\left|\theta \varphi_{0}\right|+\epsilon / 2 \leq q\left|\theta \varphi_{0}\right|+1 / 2$. We conclude $|\varphi| \leq(s+1) q$ from (10) and $q \geq 1$.

We continue with an easy lemma which gives an upper bound for $h(\varphi(p))$ if $\varphi$ : $\mathbf{G}_{m}^{n} \rightarrow \mathbf{G}_{m}^{s}$ is a homomorphism of algebraic groups and $p \in \mathbf{G}_{m}^{n}(\overline{\mathbf{Q}})$. We recall that $\varphi$ is identified with an element from $\operatorname{Mat}_{s n}(\mathbf{Z})$.

Lemma 4.3. Let $\varphi: \mathbf{G}_{m}^{n} \rightarrow \mathbf{G}_{m}^{s}$ and $p \in \mathbf{G}_{m}^{n}(\overline{\mathbf{Q}})$, then

$$
h(\varphi(p)) \leq \sqrt{s n}|\varphi| h(p) .
$$

Proof. We assume that $\varphi$ has rows $u_{i}=\left(u_{i 1}, \ldots, u_{i n}\right) \in \mathbf{Z}^{n}$ and that $p=\left(p_{1}, \ldots, p_{n}\right)$, then by the elementary height properties described in Section 2 , we deduce

$$
h(\varphi(p))=h\left(p^{u_{1}}, \ldots, p^{u_{s}}\right) \leq h\left(p^{u_{1}}\right)+\cdots+h\left(p^{u_{s}}\right) \leq \sum_{i, j}\left|u_{i j}\right| h\left(p_{j}\right) \leq\left(\sum_{i, j}\left|u_{i j}\right|\right) h(p) .
$$

The lemma follows from the Cauchy-Schwarz inequality.

Next we apply Lemma 4.3 to Lemma 4.2.

Lemma 4.4. Say $W \subset \operatorname{Mat}_{s n}(\mathbf{R})$ is an open neighborhood of $\mathcal{K}_{s n}$. Let $Q_{0}$ be the constant from Lemma 4.2 and let $Q>Q_{0}$ be a real number. If $p \in\left(\mathbf{G}_{m}^{n}\right)^{[s]}$ there exist $q \in \mathbf{Z}$ and $\varphi \in \operatorname{Mat}_{s n}(\mathbf{Z})$ such that

$$
1 \leq q \leq Q, \quad \frac{\varphi}{q} \in W, \quad h(\varphi(p)) \leq \frac{s n}{Q^{1 /(s n)}} h(p), \quad \text { and } \quad|\varphi| \leq(s+1) q
$$

Proof. Let $p \in\left(\mathbf{G}_{m}^{n}\right)^{[s]}$, then by Proposition 3.2.7 and Corollary 3.2.15 [7] there is a homomorphism of algebraic groups $\varphi_{0}: \mathbf{G}_{m}^{n} \rightarrow \mathbf{G}_{m}^{s}$ with rank $s$ and $\varphi_{0}(p)=1$. We apply Lemma 4.2 to $\varphi_{0}$, and hence obtain $q, \varphi$, and $\theta$. Accordingly, it suffices to prove the height upper bound in (11).

For brevity, we write $\delta=\varphi-q \theta \varphi_{0}$. This matrix has small norm,

$$
|\delta| \leq \frac{\sqrt{s n}}{Q^{1 /(s n)}}
$$


by (7). Since $\theta$ has rational coefficients, there is a positive integer $N$ with $N \theta \in \operatorname{Mat}_{s n}(\mathrm{Z})$. Then also $N \delta \in \operatorname{Mat}_{s n}(\mathbf{Z})$ and so

$$
(N \delta)(p)=\varphi\left(p^{N}\right)\left(q(N \theta) \varphi_{0}\right)(p)^{-1}=\varphi\left(p^{N}\right)=\varphi(p)^{N}
$$

because $\varphi_{0}(p)=1$. Taking heights and using homogeneity, we get

$$
N h(\varphi(p))=h\left(\varphi(p)^{N}\right)=h((N \delta)(p))
$$

Lemma 4.3 allows us bound

$$
h(\varphi(p))=N^{-1} h((N \delta)(p)) \leq \sqrt{s n} N^{-1}|N \delta| h(p)=\sqrt{s n}|\delta| h(p) .
$$

We get the height inequality in (11) from (12).

\section{Height Lower Bound}

The main tool used to obtain a height lower bound, which rivals the upper bound derived in Lemma 4.4, is based on a theorem of Siu. This result gives a numerical criterion to decide when the difference of two line bundles has a nonzero global section after possibly taking a large power.

A line bundle $\mathcal{L}$ on an irreducible projective variety is called numerically effective, or nef for short, if $\left(C_{1}(\mathcal{L})[C]\right) \geq 0$ for all irreducible closed curves $C$ in the said variety. For example, ample line bundles are nef, nonnegative powers of nef line bundles are nef, and by the projection formula pullbacks of nef line bundles are nef.

Theorem 5.1 (Siu). Let $X$ be an irreducible projective variety of dimension $r \geq 1$ defined over C. Say $\mathcal{L}$ and $\mathcal{M}$ are nef line bundles on $X$. If

$$
\left(c_{1}(\mathcal{L})^{r}[X]\right)>r\left(c_{1}(\mathcal{L})^{r-1} c_{1}(\mathcal{M})[X]\right),
$$

there exists a positive integer $k$ such that $\left(\mathcal{L} \otimes \mathcal{M}^{\otimes(-1)}\right)^{\otimes k}$ has a nonzero global section.

Proof. See Theorem 2.2.15, page 143 [14]. 
For the rest of this section, all varieties are defined over $\overline{\mathbf{0}}$. Let $X$ be an irreducible closed subvariety of $\mathbf{G}_{m}^{n}$ of dimension $r \geq 1$.

Proposition 5.2. Let $C_{1}$ be the constant from Lemma 3.3. Say $\varphi: \mathbf{G}_{m}^{n} \rightarrow \mathbf{G}_{m}^{r}$ is a homomorphism of algebraic groups with $\varphi \neq 0$. There exist a Zariski open and dense $U \subset X$ and a constant $C_{7}$ such that

$$
h(\varphi(p)) \geq \frac{r}{2 C_{1}}|\varphi| \frac{\Delta_{X}(\varphi)}{|\varphi|^{r}} h(p)-C_{7}
$$

for all $p \in U(\overline{\mathbf{Q}})$.

Proof. This proposition is an application of Siu's theorem and functional properties of heights associated with line bundles. We use the notation introduced in Section 3.

Since the left-hand side of (13) is nonnegative, we may assume $\Delta(\varphi)>0$. Let $\alpha$ and $\beta$ be positive integers such that

$$
\frac{1}{2} \frac{r \Delta(\varphi)}{C_{1}|\varphi|^{r-1}} \leq \frac{\beta}{\alpha}<\frac{r \Delta(\varphi)}{C_{1}|\varphi|^{r-1}}
$$

By Lemma 3.3, we estimate

$$
\begin{aligned}
\left(c_{1}\left(\bar{\varphi}^{*} \mathcal{O}(1)^{\otimes \alpha}\right)^{r-1} C_{1}\left(\pi^{*} \mathcal{O}(1)^{\otimes \beta}\right)\left[\bar{X}^{\varphi}\right]\right) & =\alpha^{r-1} \beta\left(c_{1}\left(\bar{\varphi}^{*} \mathcal{O}(1)\right)^{r-1} C_{1}\left(\pi^{*} \mathcal{O}(1)\right)\left[\bar{X}^{\varphi}\right]\right) \\
& \leq C_{1} \alpha^{r-1} \beta|\varphi|^{r-1} .
\end{aligned}
$$

We have

$$
\alpha^{r-1} \beta=\alpha^{r} \frac{\beta}{\alpha}<\alpha^{r} \frac{r \Delta(\varphi)}{C_{1}|\varphi|^{r-1}}
$$

by the second inequality in (14). Therefore,

$$
\left(c_{1}\left(\bar{\varphi}^{*} \mathcal{O}(1)^{\otimes \alpha}\right)^{r-1} C_{1}\left(\pi^{*} \mathcal{O}(1)^{\otimes \beta}\right)\left[\bar{X}^{\varphi}\right]\right)<r \alpha^{r} \Delta(\varphi) .
$$

Using Lemma 3.1(i), we can verify the hypothesis on intersection numbers in Siu's theorem,

$$
\left(c_{1}\left(\bar{\varphi}^{*} \mathcal{O}(1)^{\otimes \alpha}\right)^{r-1} C_{1}\left(\pi^{*} \mathcal{O}(1)^{\otimes \beta}\right)\left[\bar{X}^{\varphi}\right]\right)<r \alpha^{r}\left(c_{1}\left(\bar{\varphi}^{*} \mathcal{O}(1)\right)^{r}\left[\bar{X}^{\varphi}\right]\right)=r\left(c_{1}\left(\bar{\varphi}^{*} \mathcal{O}(1)^{\otimes \alpha}\right)^{r}\left[\bar{X}^{\varphi}\right]\right) .
$$


As mentioned in the beginning of this section, the line bundles $\left.\bar{\varphi}\right|_{\bar{X}^{\varphi}} ^{*} \mathcal{O}(1)^{\otimes \alpha}$ and $\left.\pi\right|_{\bar{X}^{\varphi}} ^{*} \mathcal{O}(1)^{\otimes \beta}$ are both nef. So Siu's theorem provides a positive integer $k$ such that

$$
\left(\left.\left.\bar{\varphi}\right|_{\bar{X}^{\varphi}} ^{*} \mathcal{O}(1)^{\otimes \alpha} \otimes \pi\right|_{\bar{X}^{\varphi}} ^{*} \mathcal{O}(1)^{\otimes(-\beta)}\right)^{\otimes k}
$$

has a nonzero global section.

By the properties of heights associated with line bundles discussed in Section 2, there exists $U^{\prime} \subset \bar{X}^{\varphi}$ Zariski open and dense such that if $p \in U^{\prime}(\overline{\mathbf{Q}})$, then

$$
\begin{aligned}
-C_{2} & \leq h_{\bar{X}^{\varphi},\left(\left.\left.\bar{\varphi}\right|_{\bar{X}^{\varphi}} ^{*} \mathcal{O}(1)^{\otimes \alpha} \otimes \pi\right|_{X^{\varphi}} ^{*} \mathcal{O}(1)^{\otimes(-\beta)}\right)^{\otimes k}}(p) \\
& \leq k h_{\bar{X}^{\varphi},\left.\bar{\varphi}\right|_{\bar{X}^{\varphi}} ^{*} \mathcal{O}(1)^{\otimes \alpha}}(p)-k h_{\bar{X}^{\varphi},\left.\pi\right|_{\bar{X}^{\varphi}} ^{*} \mathcal{O}(1)^{\otimes \beta}}(p)+C_{3} \\
& \leq k \alpha h_{\mathrm{P}^{r}, \mathcal{O}(1)}(\bar{\varphi}(p))-k \beta h_{\mathrm{P}^{n}, \mathcal{O}(1)}(\pi(p))+C_{4},
\end{aligned}
$$

here and in the following $C_{2}, \ldots, C_{7}$ denote constants which are independent of $p$. Now $h_{\mathbf{P}^{r}, \mathcal{O}(1)}$ and $h_{\mathbf{P}^{n}, \mathcal{O}(1)}$ differ from the absolute logarithmic Weil height on $\mathbf{P}^{r}(\overline{\mathbf{Q}})$ and $\mathbf{P}^{n}(\overline{\mathbf{Q}})$, respectively, by a bounded function. By canceling $k$ in (15), we obtain $-C_{5} \leq \alpha h(\bar{\varphi}(p))-$ $\beta h(\pi(p))+C_{6}$ on all of $U^{\prime}(\overline{\mathbf{Q}})$. Let $U=\left.b\right|_{X} ^{-1}\left(U^{\prime}\right)$, then $U$ is Zariski open and dense in $X$ and if $p \in U(\overline{\mathbf{Q}})$ we have

$$
h(\varphi(p))=h(\bar{\varphi}(b(p))) \geq \frac{\beta}{\alpha} h(\pi(b(p)))-C_{7}=\frac{\beta}{\alpha} h(p)-C_{7}
$$

on taking (2) into account. The proof follows on using the first inequality in (14).

While $C_{7}$ in this last lemma may depend on $\varphi$, it is essential that the dependency on $\varphi$ of the factor in front of $h(p)$ is completely explicit and well behaved. Later we will see lower bounds for this factor, which are uniform in $\varphi$ if $X^{\text {oa }} \neq \emptyset$.

\section{Counting Periods}

In this section, we use geometry of numbers to count periods of $\mathbf{G}_{m}^{n}$ contained in an expanding open subset of $\mathbf{C}^{n}$. All varieties are defined over $\mathbf{C}$. Let $X \subset \mathbf{G}_{m}^{n}$ be an irreducible closed subvariety of dimension $r \geq 1$. For the remainder of this section, any reference to a topology, for example, on $\mathbf{G}_{m}^{n}(\mathbf{C})$ or $X(\mathbf{C})$, will mean the usual complex topology, if not stated otherwise. 
The following lemma, which is applied here and in the next section, is classical. We give a proof for lack of reference.

Lemma 6.1. Let $Z_{1}, Z_{2}, \ldots$ be countably many Zariski closed subsets of $X$. If $Z_{1}(\mathbf{C}) \cup$ $Z_{2}(\mathbf{C}) \cup \cdots$ contains a nonempty open subset of $X(\mathbf{C})$, there exists $i$ with $X=Z_{i}$.

Proof. By the Baire category theorem, we may reduce to the case where the set of complex points of one $Z=Z_{1}$ contains a nonempty open subset $U$. Say $p \in U$, the dimensions of the complex analytic spaces $X(\mathbf{C}), U$, and $Z(\mathbf{C})$ at $p$ coincide. By GAGA, we obtain $\operatorname{dim}_{p} X=\operatorname{dim}_{p} Z$ for the corresponding varieties. But $X$ with the Zariski topology is irreducible, hence $X=Z$.

Let $\exp : \mathbf{C}^{n} \rightarrow \mathbf{G}_{m}^{n}(\mathbf{C})$ denote the $n$-fold product of the usual exponential map. It is a locally biholomorphic map between two complex manifolds and as such open.

For the remainder of this section we assume that 1 , the unit element of $\mathbf{G}_{m}^{n}$, is a nonsingular point of $X$. Now some open neighborhood $U \subset X(\mathbf{C})$ of 1 is an $r$-dimensional complex manifold. After replacing $U$ by a smaller open subset, we may assume that there is a complex manifold $M \subset \mathbf{C}^{n}$ of dimension $r$ containing 0 such that

$$
\left.\exp \right|_{M}: M \rightarrow U
$$

is biholomorphic.

We consider $\varphi \in \operatorname{Mat}_{r n}(\mathbf{C})$ as a linear map $\mathbf{C}^{n} \rightarrow \mathbf{C}^{r}$. Its restriction $\left.\varphi\right|_{M}$ is a holomorphic map between two $r$-dimensional complex manifolds. In particular, for each $z \in M$ we have a C-linear differential map

$$
d_{z}\left(\left.\varphi\right|_{M}\right): T_{z} M \rightarrow T_{\varphi(z)} \mathbf{C}^{r}=\mathbf{C}^{r}
$$

between the respective tangent spaces.

The next lemma is based on the following simple result from geometry of numbers. If $\Omega \subset \mathbf{C}^{n}$ is a discrete subgroup of rank $\rho$ and $B \subset \mathbf{C}^{n}$ is an open neighborhood of 0 , there exists a constant $C>0$, which depends on $B$ and $\Omega$, such that

$$
|\Omega \cap \lambda B| \geq C \lambda^{\rho} \quad \text { for all } \quad \lambda \geq 0
$$


Lemma 6.2. Let $\varphi_{0} \in \operatorname{Mat}_{r n}(\mathbf{C})$ such that $d_{z_{0}}\left(\left.\varphi_{0}\right|_{M}\right)$ is an isomorphism of C-vector spaces for some $z_{0} \in M$. There exist $C_{8}>0$ and an open neighborhood $W \subset \operatorname{Mat}_{r n}(\mathbf{R})$ of $\varphi_{0}$ such that

$$
\Delta_{X}(\varphi) \geq C_{8}
$$

for all $\varphi \in W \cap \operatorname{Mat}_{r n}(\mathbf{Q})$.

Proof. Consider the holomorphic map

$$
\begin{aligned}
\Psi: \operatorname{Mat}_{r n}(\mathbf{C}) \times M & \rightarrow \operatorname{Mat}_{r n}(\mathbf{C}) \times \mathbf{C}^{r}, \\
(\varphi, z) & \mapsto(\varphi, \varphi(z)) .
\end{aligned}
$$

Its differential map at $\left(\varphi_{0}, z_{0}\right)$ is an isomorphism by hypothesis. By the inverse function theorem, $\Psi$ is locally biholomorphic at $\left(\varphi_{0}, z_{0}\right)$. In particular, there exist neighborhoods $W$ and $V$ of $\varphi_{0}$ and $\varphi_{0}\left(z_{0}\right)$, respectively, such that $\Psi\left(\operatorname{Mat}_{r n}(\mathbf{C}) \times M\right) \supset W \times V$. So

$$
\text { for } \varphi \in W \text { and } y \in V \text { there is } z \in M \text { with } \varphi(z)=y \text {. }
$$

We may even assume that $V$ equals $B_{\delta}\left(\varphi_{0}\left(z_{0}\right)\right)$, an open ball in $\mathbf{C}^{r}$ around $\varphi_{0}\left(z_{0}\right)$ of radius $\delta>0$ with respect to the hermitian norm. Let $y \in B_{\delta / 2}(0)$, then

$$
V-\varphi_{0}\left(z_{0}\right)-y \supset B_{\delta / 2}(0)
$$

Let $\Omega$ be the kernel of the exponential map $\mathbf{C}^{r} \rightarrow \mathbf{G}_{m}^{r}(\mathbf{C})$, it is a discrete subgroup of rank $r$. By (16) there exists $C_{8}>0$ such that

$$
\left|\Omega \cap \lambda B_{\delta / 2}(0)\right| \geq C_{8} \lambda^{r} \text { for all } \lambda \geq 0
$$

Therefore, by (18) we have

$$
\left|\Omega \cap \lambda\left(V-\varphi_{0}\left(z_{0}\right)-y\right)\right| \geq C_{8} \lambda^{r} .
$$

Let $\varphi \in W$ and $\lambda \geq 0$, by (17) we have

$$
\lambda \varphi(M) \supset \lambda V,
$$


and so (19) implies

$$
\left|\Omega \cap \lambda\left(\varphi(M)-\varphi_{0}\left(z_{0}\right)-y\right)\right| \geq C_{8} \lambda^{r}
$$

We note that $C_{8}$ is independent of $\lambda, \varphi$, and $y$.

We now assume that $\varphi$ has rational coefficients, i.e., $\varphi \in W \cap \operatorname{Mat}_{r n}(\mathbf{0})$, and choose a positive integer $\lambda$ such that $\lambda \varphi$ has integer coefficients; we also fix $y \in B_{\delta / 2}(0)$. As observed above, there are distinct $\omega_{1}, \ldots, \omega_{N} \in \Omega$ with

$$
N \geq C_{8} \lambda^{r}
$$

such that

$$
\omega_{i}=\lambda\left(\varphi\left(z_{i}\right)-\varphi_{0}\left(z_{0}\right)-y\right)
$$

for some $z_{i} \in M$. Here, the $\omega_{i}$ and $z_{i}$ may depend on $y$. If we apply the exponential map to the equality above, we get $1=\exp \left(\omega_{i}\right)=\exp \left(\lambda \varphi\left(z_{i}\right)\right) \exp \left(\lambda \varphi_{0}\left(z_{0}\right)+\lambda y\right)^{-1}$, and so

$$
(\lambda \varphi)\left(p_{i}\right)=\exp \left(\lambda \varphi_{0}\left(z_{0}\right)+\lambda y\right)
$$

with $p_{i}=\exp \left(z_{i}\right) \in X(\mathbf{C})$ since the exponential map commutes with $\lambda \varphi \in \operatorname{Mat}_{r n}(\mathbf{Z})$.

All $p_{i}$ lie in a common fiber of $\left.(\lambda \varphi)\right|_{X}$ and they are also pairwise distinct: indeed, since $\left.\exp \right|_{M}$ is injective it suffices to show that the $z_{i}$ are pairwise distinct. Now, if $z_{i}=z_{j}$, then $\omega_{i}=\omega_{j}$ by (21), and so $i=j$ since the $\omega_{i}$ are distinct by construction.

We have shown that the fiber

$$
\left.(\lambda \varphi)\right|_{X} ^{-1}\left(\exp \left(\lambda \varphi_{0}\left(z_{0}\right)+\lambda y\right)\right)
$$

has cardinality at least $N$ for any $y \in B_{\delta / 2}(0)$. Since the exponential map is open $\exp \left(B_{\delta / 2}(0)\right) \subset \mathbf{G}_{m}^{r}(\mathbf{C})$ is open. Certainly, $\exp \left(\lambda \varphi_{0}\left(z_{0}\right)+\lambda B_{\delta / 2}(0)\right)$ is also open, and thus Zariski dense by Lemma 6.1 applied to $\mathrm{G}_{m}^{r}$. It follows from Lemma 3.1(ii) that $\Delta(\lambda \varphi) \geq N$. Inequality (20) implies $\Delta(\lambda \varphi) \geq C_{8} \lambda^{r}$. Since $\Delta(\lambda \varphi)=\lambda^{r} \Delta(\varphi)$ by homogeneity, we deduce

$$
\Delta(\varphi) \geq C_{8} \quad \text { for all } \varphi \in W \cap \operatorname{Mat}_{r n}(\mathbf{Q})
$$


We recall that $C_{8}>0$ is independent of $\varphi$ and replace $W$ by $W \cap \operatorname{Mat}_{r n}(\mathbf{R})$ to conclude the proof.

In order to prove Theorem 1.2, we shall consider morphisms $\mathbf{G}_{m}^{n} \rightarrow \mathbf{G}_{m}^{s}$ for an integer $s$ with $r \leq s \leq n$. We define $\Pi_{r s}$ to be the finite set of morphisms $\mathbf{G}_{m}^{s} \rightarrow \mathbf{G}_{m}^{r}$ defined by projecting to $r$ distinct coordinates of $\mathbf{G}_{m}^{s}$. As usual, elements of $\Pi_{r s}$ are identified with elements of $\mathrm{Mat}_{r s}(\mathrm{Z})$. The next lemma relies on a simple compactness argument.

Lemma 6.3. Let $\mathcal{K} \subset \mathrm{Mat}_{\text {sn }}(\mathbf{R})$ be compact. One of the following cases holds:

(i) There exists $\varphi_{0} \in \mathcal{K}$ such that for all $z \in M$ the differential

$$
d_{z}\left(\left.\varphi_{0}\right|_{M}\right): T_{Z} M \rightarrow \mathbf{C}^{s}
$$

is not injective.

(ii) There exists $C_{9}>0$ and an open neighborhood $W \subset \operatorname{Mat}_{s n}(\mathbf{R})$ of $\mathcal{K}$ such that for each $\varphi \in W \cap \operatorname{Mat}_{s n}(\mathbf{Q})$ there is $\pi \in \Pi_{r s}$ with $\Delta_{X}(\pi \varphi) \geq C_{9}$.

Proof. We will assume that case (i) does not hold and will show that case (ii) does.

Let $\varphi_{0} \in \mathcal{K}$. There exist $\pi \in \Pi_{r s}$ and $z \in M$ such that $d_{z}\left(\left.\pi \varphi_{0}\right|_{M}\right)$ is injective, and hence an isomorphism of C-vector spaces. By Lemma 6.2, we may find an open neighborhood of $\pi \varphi_{0}$ in $\mathrm{Mat}_{r n}(\mathbf{R})$ with the stated properties. It follows that we may find $W_{\varphi_{0}}$, an open neighborhood of $\varphi_{0}$ in $\operatorname{Mat}_{s n}(\mathbf{R})$, and $C_{\varphi_{0}}>0$ with $\Delta(\pi \varphi) \geq C_{\varphi_{0}}$ for all $\varphi \in W_{\varphi_{0}} \cap \operatorname{Mat}_{s n}(\mathbf{Q})$.

The open cover $\bigcup_{\varphi_{0} \in \mathcal{K}} W_{\varphi_{0}}$ contains $\mathcal{K}$. Since $\mathcal{K}$ is compact, we may pass to a finite subcover and conclude that there exist $C_{9}>0$ and an open subset $W$ of $\operatorname{Mat}_{s n}(\mathbf{R})$ containing $\mathcal{K}$ such that for each $\varphi \in W \cap \operatorname{Mat}_{s n}(\mathbf{Q})$ there is $\pi \in \Pi_{r s}$ with $\Delta(\pi \varphi) \geq C_{9}$.

\section{Applying Ax's Theorem}

In Proposition 7.3, the main result of this section, we apply a theorem of Ax to replace case (i) in Lemma 6.3 by a condition connected to the set $X^{\mathrm{oa},[s]}$ defined in the introduction.

All varieties in this section will be defined over C. Let $X \subset \mathrm{G}_{m}^{n}$ be an irreducible closed subvariety of dimension $r \geq 1$. Furthermore, let $s$ be an integer with $r \leq s \leq n$. Unless stated otherwise, any reference to a topology in this section will mean the complex topology.

Before proving Proposition 7.3, we need a lemma. 
Lemma 7.1. Let $H \subset \mathbf{G}_{m}^{n}$ be an algebraic subgroup and let $k \in \mathbf{Z}$, then

$$
Z(H, k)=\left\{p \in X(\mathbf{C}) ; \operatorname{dim}_{p} X \cap p H \geq k\right\}
$$

is Zariski closed in $X$.

Proof. By Corollary 3.2.15 [7], there is an morphism of algebraic groups $\varphi: \mathbf{G}_{m}^{n} \rightarrow \mathbf{G}_{m}^{n^{\prime}}$ whose kernel is precisely $H$. Then we have $Z(H, k)=\left\{p \in X(\mathbf{C}) ;\left.\operatorname{dim}_{p} \varphi\right|_{X} ^{-1}(\varphi(p)) \geq k\right\}$ and the lemma follows from semicontinuity theorem of Chevalley (second theorem on page 228 [9]).

The following theorem is a consequence of Ax's theorem 1 ([2], see also Corollary 1). Ax's original result applies to general algebraic groups over $\mathbf{C}$.

Theorem 7.2 (Ax). Let $A$ be a connected analytic subgroup of $\mathbf{G}_{m}^{n}(\mathbf{C})$, i.e., the image of a C-vector space under the exponential map. Let $K$ be an irreducible analytic subvariety of an open subset of $\mathbf{G}_{m}^{n}(\mathbf{C})$ with $1 \in K$ and $K \subset A$. If $V$ is the Zariski closure of $K$, there exists an algebraic subgroup $H \subset \mathbf{G}_{m}^{n}$ containing $V$ with $\operatorname{dim} H \leq \operatorname{dim} V+\operatorname{dim} A-\operatorname{dim} K$.

This result is applied in the following proposition.

Proposition 7.3. Let $\mathcal{K} \subset \operatorname{Mat}_{s n}(\mathbf{R})$ be compact and such that all its elements have rank $s$. One of the following cases holds:

(i) There exists an algebraic subgroup $H \subset \mathbf{G}_{m}^{n}$ such that

$$
\operatorname{dim}_{p} X \cap p H \geq \max \{1, s+\operatorname{dim} H-n+1\}
$$

for all $p \in X(\mathbf{C})$.

(ii) There exists $C_{10}>0$ and an open neighborhood $W \subset \operatorname{Mat}_{s n}(\mathbf{R})$ of $\mathcal{K}$ such that for each $\varphi \in W \cap \operatorname{Mat}_{s n}(\mathbf{Q})$ there is $\pi \in \Pi_{r s}$ with $\Delta_{X}(\pi \varphi) \geq C_{10}$.

Proof. It follows from Lemma 3.1(ii) that the function $\Delta$ is invariant under translation of $X$. Hence, it is no restriction to assume that 1 is a nonsingular point of $X$ as in Section 6. Moreover, we let $U$ and $M$ be as in said section, so $M \subset \mathbf{C}^{n}$ and $U \subset X(\mathbf{C})$ are complex manifolds with $\left.\exp \right|_{M}: M \rightarrow U$ biholomorphic and $U$ is open in $X(\mathbf{C})$ containing 1 . 
If case (ii) of Lemma 6.3 holds, then case (ii) of this lemma holds. Hence, we may assume that we are in case (i) of Lemma 6.3; we will show that case (i) of this lemma holds.

Let us assume there exists $\varphi_{0} \in \mathcal{K}$ such that for all $z \in M$ the differential $d_{z}\left(\left.\varphi_{0}\right|_{M}\right)$ fails to be injective. We consider $\operatorname{ker} \varphi_{0}$, the kernel of $\varphi_{0}$, as a $\mathbf{C}$-vector subspace of $\mathbf{C}^{n}$. It follows from Corollary 7F, page 314 [19] that after replacing $M$ by a nonempty open subset, the fiber

$$
\left.\varphi_{0}\right|_{M} ^{-1}\left(\varphi_{0}(z)\right)=\left(z+\operatorname{ker} \varphi_{0}\right) \cap M
$$

is a complex submanifold of $M$ with positive dimension for any $z \in M$; we may still assume $U=\exp (M)$. By our hypothesis on $\mathcal{K}$ we have $\operatorname{dim} \operatorname{ker} \varphi_{0}=n-s$ and so the connected analytic subgroup $A=\exp \left(\operatorname{ker} \varphi_{0}\right) \subset \mathbf{G}_{m}^{n}(\mathbf{C})$ has dimension $n-s$. Applying the exponential map, we see that for each $p \in \exp (M)$ the intersection

$$
A \cap p^{-1} X(\mathbf{C})
$$

contains a connected complex submanifold $K_{p}$ of an open subset of $\mathbf{G}_{m}^{n}(\mathbf{C})$ with $\operatorname{dim} K_{p} \geq 1$ and $1 \in K_{p}$.

Let $V_{p} \subset \mathbf{G}_{m}^{n}$ be the Zariski closure of $K_{p}$. It is certainly contained in $p^{-1} X$ and is irreducible in the Zariski topology by the theorem on page 168 [11]. By Ax's theorem, $V_{p}$ is contained in an algebraic subgroup $H_{p}$ with $\operatorname{dim} H_{p} \leq \operatorname{dim} V_{p}+\operatorname{dim} A-\operatorname{dim} K_{p}$. Since $\operatorname{dim} V_{p} \geq \operatorname{dim} K_{p} \geq 1$ we have $\operatorname{dim} V_{p} \geq \max \left\{1, \operatorname{dim} H_{p}+s-n+1\right\}$, and hence

$$
p \in Z\left(H_{p}, \max \left\{1, \operatorname{dim} H_{p}+s-n+1\right\}\right)
$$

using the notation introduced in (22).

Because the above statement holds for all $p \in \exp (M)=U$, we conclude

$$
U \subset \bigcup_{H} Z(H, \max \{1, \operatorname{dim} H+s-n+1\}),
$$

where the union is taken over all algebraic subgroups $H \subset \mathbf{G}_{m}^{n}$. By Lemma 7.1, each set in the union on the right of (23) is Zariski closed in $X$. By construction $U \subset X(\mathbf{C})$ is open and nonempty. Finally, $\mathbf{G}_{m}^{n}$ only has countably many algebraic subgroups, and so the proof follows from Lemma 6.1. 
In our application, we will take $\mathcal{K}$ to equal $\mathcal{K}_{s n}$, the set defined in Section 4 . We note that case (i) of this proposition implies $X^{\mathrm{oa},[s]}=\emptyset$.

\section{Proof of the Bounded Height Theorem and Corollaries 1.4 and 1.5}

All varieties in this section are assumed to be defined over $\overline{\mathbf{Q}}$. We set $G=\mathbf{G}_{m}^{n}$. For the moment, let $X$ be an irreducible closed subvariety of $G$ of dimension $r \geq 1$.

Before proceeding further, we make the following easy observation: say $s$ is an integer and $0<\epsilon \leq 1 /(2 n)$ with $p \in \mathcal{C}\left(G^{[s]}, \epsilon\right)$, so there is $a \in G^{[s]}$ and $b \in G(\overline{\mathbf{0}})$ with $p=$ $a b$ and $h(b) \leq \epsilon(1+h(a))$. Then $h(a)=h\left(p b^{-1}\right) \leq h(p)+h\left(b^{-1}\right) \leq h(p)+n h(b) \leq h(p)+(1+$ $h(a)) / 2$ by the elementary height properties stated in Section 2 . We easily deduce

$$
h(a) \leq 1+2 h(p) \text { and } h(b) \leq 2 \epsilon(1+h(p)) .
$$

Lemma 8.1. Let $Y \subset X$ be an irreducible closed subvariety of positive dimension and let $s$ be an integer with $\operatorname{dim} Y \leq s \leq n$. If $Y \cap X^{\mathrm{oa},[s]} \neq \emptyset$, there exist $\epsilon>0$ and $U \subset Y$ which is Zariski open and dense such that the height is bounded from above on $U(\overline{\mathbf{Q}}) \cap \mathcal{C}\left(G^{[s]}, \epsilon\right)$.

Proof. The condition $Y \cap X^{\mathrm{oa},[s]} \neq \varnothing$ implies that we are in case (ii) of Proposition 7.3 applied to $Y$ and $\mathcal{K}=\mathcal{K}_{s n}$, the latter set was defined in Section 4 . Therefore, there exists an open set $W \subset \operatorname{Mat}_{s n}(\mathbf{R})$ containing $\mathcal{K}_{s n}$ and $C_{10}>0$ such that for each $\varphi \in W \cap \operatorname{Mat}_{s n}(\mathbf{Q})$ there is $\pi \in \Pi_{\operatorname{dim} Y, s}$ with $\Delta_{Y}(\pi \varphi) \geq C_{10}$.

We suppose $Q_{0}$ is as in Lemma 4.4 and that $Q>Q_{0}$ is a fixed parameter and depends only on $X$ and $Y$. We will see later how to choose $Q$ properly.

Let $\Phi$ denote the set of all matrices $\varphi \in \operatorname{Mat}_{s n}(\mathbf{Z})$ such that there exists an integer $q$ with $1 \leq q \leq Q, \varphi / q \in W$, and $|\varphi| \leq(s+1) q$ (see Lemma 4.4). Clearly, $\Phi$ is a finite set.

For each $\varphi \in \Phi$, there is a $\pi \in \Pi_{\operatorname{dim} Y, s}$ such that $\Delta_{Y}\left(\varphi^{\prime} / q\right) \geq C_{10}$ where $\varphi^{\prime}=\pi \varphi$. In particular, $\varphi^{\prime} \neq 0$ since $C_{10}>0$. By homogeneity we have

$$
\Delta_{Y}\left(\varphi^{\prime}\right)=q^{\operatorname{dim} Y} \Delta_{Y}\left(\varphi^{\prime} / q\right) \geq C_{10} q^{\operatorname{dim} Y}
$$

Now $\varphi^{\prime} \neq 0$ implies $\left|\varphi^{\prime}\right| \geq 1$ so we obtain the following lower bound for the factor in front of $h(p)$ in (13):

$$
C_{11}\left|\varphi^{\prime}\right| \frac{\Delta_{Y}\left(\varphi^{\prime}\right)}{\left|\varphi^{\prime}\right|^{\operatorname{dim} Y}} \geq C_{10} C_{11}\left|\varphi^{\prime}\right| \frac{q^{\operatorname{dim} Y}}{\left|\varphi^{\prime}\right|^{\operatorname{dim} Y}} \geq C_{10} C_{11} \frac{q^{\operatorname{dim} Y}}{\left|\varphi^{\prime}\right|^{\operatorname{dim} Y}}
$$


with

$$
C_{11}=\frac{\operatorname{dim} Y}{2(4 n)^{\operatorname{dim} Y} \operatorname{deg}(Y)}>0
$$

Because $\left|\varphi^{\prime}\right| \leq|\varphi| \leq(s+1) q$ we have

$$
C_{11}\left|\varphi^{\prime}\right| \frac{\Delta_{Y}\left(\varphi^{\prime}\right)}{\left|\varphi^{\prime}\right|^{\operatorname{dim} Y}} \geq \frac{C_{10} C_{11}}{(s+1)^{\operatorname{dim} Y}}
$$

We denote this last quantity by $C_{12}$; it is positive and independent of $Q$ and $\varphi \in \Phi$.

We fix

$Q=\max \left\{Q_{0}+1,\left(8 s n C_{12}^{-1}\right)^{s n}\right\}>Q_{0} \quad$ and $\quad \epsilon=\min \left\{\frac{1}{2 n}, \frac{\sqrt{s n}}{s+1} \frac{1}{Q^{1+1 /(s n)}}\right\} \in\left(0, \frac{1}{2 n}\right]$

Let $U_{\varphi}$ be the Zariski open and dense subset of $Y$ supplied by Proposition 5.2 applied to $\varphi^{\prime}$. The intersection

$$
U=\bigcap_{\varphi \in \Phi} U_{\varphi}
$$

is Zariski open and dense in $Y$ since $\Phi$ is finite. We deduce that

$$
h\left(\varphi^{\prime}(p)\right) \geq C_{12} h(p)-C(Q) \text { for all } p \in U(\overline{\mathbf{Q}}) \text { and all } \varphi \in \Phi,
$$

here $C(Q)$ depends neither on $p$ nor on $\varphi$ (but possibly on $Q$ ).

Now let us assume that $p \in U(\overline{\mathbf{Q}}) \cap \mathcal{C}\left(G^{[s]}, \epsilon\right)$, that is, there are $a \in G^{[s]}$ and $b \in G(\overline{\mathbf{Q}})$ with $p=a b$ and $h(b) \leq \epsilon(1+h(a))$.

By Lemma 4.4, there exists $\varphi \in \Phi$ with $h(\varphi(a)) \leq \operatorname{sn} Q^{-1 /(s n)} h(a)$ and so

$$
h(\varphi(a)) \leq 2 \operatorname{sn} Q^{-1 /(s n)}(1+h(p))
$$

by (24).

We apply Lemma 4.3 in order to bound $h(\varphi(b)) \leq \sqrt{s n}|\varphi| h(b)$. Now (24) gives $h(\varphi(b)) \leq 2 \epsilon \sqrt{s n}|\varphi|(1+h(p))$. But $|\varphi| \leq(s+1) q \leq(s+1) Q$, so

$$
h(\varphi(b)) \leq 2 \epsilon \sqrt{\operatorname{sn}}(s+1) Q(1+h(p))
$$


Using an elementary height property and (27), (28) gives

$$
\begin{aligned}
h(\varphi(p)) & =h(\varphi(a b)) \leq h(\varphi(a))+h(\varphi(b)) \\
& \leq\left(2 s n Q^{-1 /(s n)}+2 \epsilon \sqrt{s n}(s+1) Q\right)(1+h(p)) .
\end{aligned}
$$

The choice of $\epsilon$ made in (25) implies $h(\varphi(p)) \leq 4 \operatorname{sn} Q^{-1 /(s n)}(1+h(p))$ and the choice of $Q$ gives $h(\varphi(p)) \leq C_{12}(1+h(p)) / 2$. Furthermore, we have $h\left(\varphi^{\prime}(p)\right) \leq h(\varphi(p))$, hence

$$
h\left(\varphi^{\prime}(p)\right) \leq \frac{C_{12}}{2}(1+h(p))
$$

If we compare (26) and (29), we immediately get the desired $h(p) \leq 1+2 C_{12}^{-1} C(Q)$.

For brevity, we set $\Sigma=X^{\mathrm{oa},[s]} \subset X(\overline{\mathbf{Q}})$. If $\Sigma \neq \emptyset$, then Lemma 8.1 applied with $X=Y$ shows that there exists $U \subset X$ Zariski open and dense such that $U(\overline{\mathbf{Q}}) \cap G^{[s]}$ has bounded height. This is already close to Theorem 1.2, and the following simple argument shows how to deal with the points in $(\Sigma \backslash U(\overline{\mathbf{Q}})) \cap G^{[s]}$.

Lemma 8.2. Let $s$ be an integer with $r \leq s \leq n$ and say there is a proper subset $S \subsetneq \Sigma$ and an $\epsilon>0$ such that the height is bounded from above on $S \cap \mathcal{C}\left(G^{[r]}, \epsilon\right)$. There exists a subset $S^{\prime} \subset \Sigma$ containing $S$ with $\overline{\Sigma \backslash S^{\prime}} \subsetneq \overline{\Sigma \backslash S}$ and an $\epsilon^{\prime}>0$ such that the height is bounded from above on $S^{\prime} \cap \mathcal{C}\left(G^{[r]}, \epsilon^{\prime}\right)$.

Proof. By assumption $\Sigma \backslash S$ is nonempty. Hence, its Zariski closure $\overline{\Sigma \backslash S}$ has an irreducible component $Y$ with $Y \cap \Sigma \neq \emptyset$; in particular, $\overline{\Sigma \backslash S}=Y \cup Z$ with $Z$ Zariski closed and $Y \not \subset Z$. If $Y$ has positive dimension we may apply Lemma 8.1 and find a Zariski open dense $U \subset Y$ and $\epsilon^{\prime}>0$ such that the height is bounded from above on $U(\overline{\mathbf{Q}}) \cap \mathcal{C}\left(G^{[s]}, \epsilon^{\prime}\right)$. If $Y$ is a point, then the existence of $U$ and $\epsilon^{\prime}$ is obvious.

Clearly, we may assume $\epsilon^{\prime} \leq \epsilon$. We set $S^{\prime}=S \cup(U(\overline{\mathbf{Q}}) \cap \Sigma)$. By hypothesis and the previous paragraph the height is bounded from above on $S^{\prime} \cap \mathcal{C}\left(G^{[s]}, \epsilon^{\prime}\right)$. Of course $\Sigma \backslash S^{\prime} \subset(Y \backslash U) \cup Z$ and even $\overline{\Sigma \backslash S^{\prime}} \subset(Y \backslash U) \cup Z$. So $\overline{\Sigma \backslash S^{\prime}}=\overline{\Sigma \backslash S}$ is impossible and the lemma follows.

Proof of Theorem 1.2: Let $X$ be as in the hypothesis. We may assume $\operatorname{dim} X \geq 1, s \geq \operatorname{dim} X$ because otherwise $X^{\mathrm{oa},[s]}=\emptyset$, and finally $s \leq n$. We set $S_{0}=\emptyset$ and $\epsilon_{0}=1$. Let $k \geq 1$. By induction we assume $S_{k-1}$ is a subset of $\Sigma$ and $\epsilon_{k-1}>0$ such that $S_{k-1} \cap \mathcal{C}\left(G^{[s]}, \epsilon_{k-1}\right)$ has 
bounded height. The theorem follows if $S_{k-1}$ equals $\Sigma$, so let us assume $S_{k-1} \subsetneq \Sigma$. In this case we apply Lemma 8.2 to find a subset $S_{k} \subset \Sigma$ containing $S_{k-1}$ and an $\epsilon_{k}>0$ such that the height is bounded on $S_{k} \cap \mathcal{C}\left(G^{[s]}, \epsilon_{k}\right)$.

We obtain a chain

$$
X \supset \overline{\Sigma \backslash S_{0}} \supsetneq \overline{\Sigma \backslash S_{1}} \supsetneq \cdots \supsetneq \overline{\Sigma \backslash S_{k}} .
$$

But $X$, being a noetherian topological space, satisfies the descending chain condition for Zariski-closed sets. In our situation, this means $S_{k}=\Sigma$ for some $k$, the theorem follows with $\epsilon=\epsilon_{k}$.

Corollary 1.4 follows immediately from Corollary 1.3 and the following result by Bombieri, Masser, and Zannier.

Theorem 8.3 (Bombieri, Masser, Zannier, Lemma 8.1 [5]). Let $G=\mathrm{G}_{m}^{n}$ and let $X \subset G$ be an irreducible closed subvariety defined over $\overline{\mathbf{Q}}$. If $B \in \mathbf{R}$ then $\left\{p \in X^{\mathrm{ta}} \cap G^{[1+\operatorname{dim} X]}\right.$; $h(p) \leq B\}$ is finite.

We remark that although we have not stated the definition of $X^{\text {ta }}$, it can be found in the paper just cited, the above theorem holds with $X^{\text {ta }}$ replaced by $X^{\text {oa }}$ since $X^{\text {oa }} \subset X^{\text {ta }}$.

Finally, we come to the proof of Corollary 1.5. We know from the work of Bombieri, Masser, and Zannier (Theorem 1.4(b) [4]) that $X^{\text {oa }}$ is Zariski open in $X$, so by virtue of Corollary 1.4 it suffices to show $X^{\text {oa }} \neq \emptyset$. But Theorem 1.4(b) even gives a structural description of $X^{\mathrm{oa}}$; in particular if this set is empty, it shows that there are monomials as in (1) which, as elements of the function field of $X$, are algebraically dependent over $\overline{\mathbf{Q}}$.

\section{Acknowledgements}

I would like to thank David Masser for pointing out to me Ax's work [2].

\section{References}

[1] Amoroso, F., and S. David. "Distribution des points de petite hauteur dans les groupes multiplicatifs." Annali della Scuola Normale Superiore di Pisa-Classe di Scienze (5), 3(2) (2004): 325-48.

[2] Ax, James. "Some topics in differential algebraic geometry I: Analytic subgroups of algebraic groups." American Journal of Mathematics 94 (1972): 1195-1204. 
[3] Bombieri, E., D. Masser, and U. Zannier. "Intersecting a curve with algebraic subgroups of multiplicative groups." International Mathematics Research Notices 20 (1999): 1119-40.

[4] Bombieri, E., D. Masser, and U. Zannier. "Anomalous subvarieties: Structure theorems and applications." International Mathematics Research Notices 19 (2007): 1-33.

[5] Bombieri, E., D. Masser, and U. Zannier. "Intersecting a plane with algebraic subgroups of multiplicative groups." Annali della Scuola Normale Superiore di Pisa-Classe di Scienze (5), 7 (2008): 51-80.

[6] Bombieri, E., D. Masser, and U. Zannier. “On unlikely intersections of complex varieties with tori." Acta Arithmetica (forthcoming).

[7] Bombieri, Enrico, and Walter Gubler. Heights in Diophantine Geometry. Cambridge: Cambridge University Press, 2006.

[8] Cassels, J. W. S. An Introduction to Diophantine Approximation. Cambridge: Cambridge University Press, 1957.

[9] Danilov, V. I. "Algebraic varieties and schemes." In Algebraic Geometry I, edited by I. R. Shafarevich, Encyclopaedia of Mathematical Sciences 23. Berlin: Springer, 1994.

[10] Fulton, William. Intersection Theory. Berlin: Springer, 1984.

[11] Grauert, H., and R. Remmert. Coherent Analytic Sheaves. Berlin: Springer, 1984.

[12] Habegger, P. "Intersecting subvarieties of abelian varieties with algebraic subgroups of complementary dimension." Inventiones Mathematicae (forthcoming).

[13] Habegger, P. "Intersecting subvarieties of $\mathrm{G}_{m}^{n}$ with algebraic subgroups." Mathematische Annalen 342(2) (2008): 449-66.

[14] Lazarsfeld, R. Positivity in Algebraic Geometry I. Berlin: Springer, 2004.

[15] Philippon, Patrice. "Lemmes de zéros dans les groupes algébriques commutatifs." Bulletin de la Société Mathématique de France 114 (1986): 355-83.

[16] Pink, Richard. "A common generalization of the conjectures of André-Oort, Manin-Mumford, and Mordell-Lang." (2005): preprint.

[17] Rémond, Gaël. "Intersection de sous-groupes et de sous-variétés 3." Commentarii Mathematici Helvetici (forthcoming).

[18] Schinzel, A. "Polynomials with special regard to reducibility. With an appendix by Umberto Zannier." Encyclopedia of Mathematics and its Applications, vol. 77. Cambridge: Cambridge University Press, 2000.

[19] Whitney, Hassler. Complex Analytic Varieties. Philippines: Addison-Wesley, 1972.

[20] Zannier, Umberto. "Appendix by Umberto Zannier in [18] (517-39)." Encyclopedia of Mathematics and its Applications, vol. 77. Cambridge: Cambridge University Press, 2000.

[21] Zilber, Boris. "Exponential sums equations and the Schanuel conjecture." Journal of the London Mathematical Society 2, 65(1) (2002): 27-44. 